\title{
BMJ Open One- and two-year visual outcomes from the Moorfields age-related macular degeneration database: a retrospective cohort study and an open science resource
}

\author{
Katrin Fasler, ${ }^{01,2}$ Gabriella Moraes, ${ }^{1}$ Siegfried Wagner, ${ }^{1}$ Karsten U Kortuem, ${ }^{1,3}$ \\ Reena Chopra, ${ }^{1}$ Livia Faes, ${ }^{1,4}$ Gabriella Preston, ${ }^{1}$ Nikolas Pontikos, ${ }^{1}$ Dun Jack Fu, ${ }^{1}$ \\ Praveen Patel, ${ }^{1}$ Adnan Tufail, ${ }^{1}$ Aaron Y Lee, ${ }^{5}$ Konstantinos Balaskas, ${ }^{1,6}$ \\ Pearse A Keane ${ }^{1}$
}

To cite: Fasler K, Moraes G, Wagner $\mathrm{S}$, et al. One- and two-year visual outcomes from the Moorfields agerelated macular degeneration database: a retrospective cohort study and an open science resource. BMJ Open 2019;9:e027441. doi:10.1136/ bmjopen-2018-027441

- Prepublication history and additional material for this paper are available online. To view please visit the journal (http:// dx.doi.org/10.1136/bmjopen2018-027441).

Received 29 0ctober 2018 Revised 16 April 2019 Accepted 20 May 2019
Check for updates

(C) Author(s) (or their employer(s)) 2019. Re-use permitted under CC BY-NC. No commercial re-use. See rights and permissions. Published by BMJ.

For numbered affiliations see end of article.

Correspondence to Dr Pearse A Keane; pearse.keane1@nhs.net

\section{ABSTRACT}

Objectives To analyse treatment outcomes and share clinical data from a large, single-centre, well-curated database (8174 eyes/6664 patients with 120756 single entries) of patients with neovascular age-related macular degeneration (AMD) treated with anti-vascular endothelial growth factor (VEGF). By making our depersonalised raw data openly available, we aim to stimulate further research in AMD, as well as set a precedent for future work in this area.

Setting Retrospective, comparative, non-randomised electronic medical record (EMR) database cohort study of the UK Moorfields AMD database with data extracted between 2008 and 2018.

Participants Including one eye per patient, 3357 eyes/patients (61\% female). Extraction criteria were $\geq 1$ ranibizumab or aflibercept injection, entry of 'AMD' in the diagnosis field of the EMR and a minimum of 1 year of follow-up. Exclusion criteria were unknown date of first injection and treatment outside of routine clinical care at Moorfields before the first recorded injection in the database.

Main outcome measures Primary outcome measure was change in VA at 1 and 2 years from baseline as measured in Early Treatment Diabetic Retinopathy Study letters. Secondary outcomes were the number of injections and predictive factors for VA gain.

Results Mean VA gain at 1 year and 2 years were +5.5 ( $95 \% \mathrm{Cl} 5.0$ to 6.0 ) and +4.9 (95\% Cl 4.2 to 5.6 ) letters, respectively. Fifty-four per cent of eyes gained $\geq 5$ letters at 2 years, $63 \%$ had stable VA ( $\pm \leq 14$ letters), $44 \%$ of eyes maintained good VA ( $\geq 70$ letters). Patients received a mean of 7.7 (95\% $\mathrm{Cl} 7.6$ to 7.8$)$ injections during year 1 and 13.0 (95\% $\mathrm{Cl} 12.8$ to 13.2) injections over 2 years. Younger age, lower baseline VA and more injections were associated with higher VA gain at 2 years.

Conclusion This study benchmarks high quality EMR study results of real life AMD treatment and promotes open science in clinical AMD research by making the underlying data publicly available.

\section{Strengths and limitations of this study}

- Large sample size, retrospective, single centre, electronic medical record database study

- High-quality real life data

Open science approach with sharing of depersonalised raw data

\section{INTRODUCTION}

The treatment of neovascular age-related macular degeneration (AMD) has been revolutionised by the development of anti-vascular endothelial growth factor (VEGF) agents such as ranibizumab and aflibercept. ${ }^{1-4}$ Unfortunately, real world results from retrospective studies are typically inferior to those from randomised controlled trials (RCTs), with fewer administered injections and significant intercountry and intercentre differences in therapy administration and outcomes. $^{5-9}$ Although retrospective studies and audits may be more likely than RCTs to reflect results in clinical practice, they still are not truly representative of outcomes in real world populations. ${ }^{5-71011}$ Major drawbacks of retrospective study designs are small sample sizes with selection bias and suboptimal methods for handling of both missing data and losses to follow-up (LTFU). ${ }^{11} 12$ Survival bias in particular can lead to skewed results: omission of cases LTFU from the analysis leads to selection of a non-random cohort with potential overestimation of visual acuity (VA) gains through exclusion of patients that stop treatment early due to irreversible visual loss such as foveal scarring or other adverse effects. $^{12}$ 
The advent of electronic medical records (EMR) has facilitated the collection of large amounts of data in routine clinical practice and thus has the potential to make retrospective study populations more representative of real life. ${ }^{13-18}$ This is very much dependent, however, on the quality of data entry and the reliable follow-up of patients, and so these issues can remain problematic. The amount of data available from EMR systems also challenges the traditional methods of validation, analytics and reporting, and there is a struggle to implement the existing clinical research guidelines. ${ }^{12}{ }^{19-21}$ For example, in 2015, the RECORD statement highlighted the challenges of using routinely collected observational health data. ${ }^{21} \mathrm{~A}$ further problem is the variation of data collection in the different EMR registers in different hospitals and countries. The International Consortium for Health Outcomes Measurement (ICHOM) AMD working group has also proposed a standard set of clinical characteristics, interventions and outcomes including preferential methods of VA recording (logarithm of the minimum angle of resolution or Early Treatment Diabetic Retinopathy Study (ETDRS) letters) ${ }^{22}$

At Moorfields Eye Hospital, an EMR was initiated in October 2008, and its successor, OpenEyes, was implemented in September 2012. Subsequently, data from both systems were merged into the current centralised repository, the data warehouse. We have created a data set from this which represents, to our knowledge, the largest single-centre cohort of patients receiving treatment for neovascular AMD in the world. This Moorfields AMD data set is increasing steadily, with 909 new patients in 2017 alone, a number typically only comparable in magnitude to multicentre studies. ${ }^{14} 16$ Apart from its sheer size, key advantages of this data set include the ability to clean and validate data directly, the completeness due to the mandatory input of relevant fields including VA, the consistency of VA measurements in ETDRS letters, the lack of requirement to merge data from different sites and systems, the standardised treatment scheme following national guidelines, and the ability to directly access the raw imaging data from each study visit.

The aim of this study is to analyse 1-year and 2-year VA outcomes, determine predictive factors of VA gain in treatment-naive eyes from the Moorfields AMD database and to aid in scientific progress by making the depersonalised raw data from our study openly available to the research community. ${ }^{21} 23$

\section{METHODS}

\section{Data collection}

Data for this retrospective, comparative, non-randomised cohort study were extracted from the data warehouse, the centralised storage for all EMR data, of Moorfields Eye Hospital. Data were extracted between 21 October 2008 and 8 August 2018. Extraction criteria were $\geq 1$ ranibizumab or aflibercept injection, entry of 'AMD' in the diagnosis field of the EMR and a minimum of 1 year of follow-up. Exclusion criteria were unknown date of first injection, any treatment outside of routine clinical care at Moorfields before the first recorded injection in the database, including pegaptanib, previous laser or photodynamic therapy, and bevacizumab. The rationale for exclusion of bevacizumab is that in the National Health Service (NHS), neovascular AMD is generally treated with the licensed therapeutics ranibizumab or aflibercept, and not with the off-label bevacizumab. ${ }^{245}$ The date of the first injection was defined as the baseline date. The data set has been depersonalised for publication and approval for data collection and analysis was obtained from the Institutional Review Board at Moorfields (ROAD17/031). The study adhered to the tenets set forth in the Declaration of Helsinki.

\section{Outcome measures}

The primary outcomes were mean change in VA from baseline as measured in ETDRS letters, proportion of eyes gaining $\geq 5$ letters, proportion of eyes with stable vision (change in $\mathrm{VA}<15$ letters to baseline), proportion of eyes with good vision ( $\geq 20 / 40$ or 70 letters), and proportion of eyes with poor vision ( $\leq 20 / 200$ or 35 letters) at baseline, year one, and year two. Those endpoints have been used in the pivotal trials and/or have been included in the ICHOM reporting recommendations. ${ }^{1-4} 22$ Secondary outcomes included the number of injections, and effect of baseline characteristics and injection numbers on changes in VA. Definitions for 1 year and 2 year outcome dates were taken from previous real-world studies as visits closest to 52 weeks and 104 weeks post baseline date within \pm 8 weeks. ${ }^{613}$ We used the STROBE cohort checklist when writing our report. ${ }^{20}$

\section{Efforts to minimise bias}

Clinical information from patients with neovascular AMD is manually entered to the Moorfields Eye Hospital EMR (OpenEyes) at each visit. The EMR requires mandatory completion for a number of fields at each patient visit, including VA, central retinal thickness, treatment decision, treatment drug and injection number, thus minimising the number of missing data entries. Of all 120756 single entries, missing/zerovisual acuity measurements were encountered in 4059 (4.1\%) of all entries. After manual cleaning of all 4059 missing entries, missing data accounted for $808(0.9 \%)$ entries. Patients aged $<55$ or $>100$ and eyes with injection numbers $\geq 50$ were manually checked. Description of manual cleaning including a CONSORT diagram is shown in supplementary material (see online supplementary 1, sFigure 1). Visual acuities below measurable ETDRS letters were converted to $\log$ MAR 2.0/-15 letters, logMAR 2.3/-30 letters and $\log$ MAR $2.7 /-50$ letters for count fingers, hand movements and light perception, respectively. ${ }^{26}$ To avoid bias due to intereye correlation, statistical analysis was restricted to one eye per patient, that is, the first eye of a patient if sequentially treated, and a randomly selected 
eye if simultaneously treated. Outcomes of second-treated fellow eyes will be reported separately.

\section{Statistical analysis}

The data were analysed using the statistics software $\mathrm{R}$ (https://www.r-project.org/; provided in the public domain by R Core team 2017 R Foundation for Statistical Computing, Vienna, Austria). The ggplot2 package was used for plots. The eye was defined as unit of analysis. Descriptive statistics included mean $+/-95 \%$ CI and median, where appropriate. Differences between groups were evaluated using Mann Whitney $\mathrm{U}$ test and Pearson chi-square. Multivariate linear regression analysis was performed to assess relationship of predictive factors and VA change. Independent variables used included gender, baseline age, baseline VA and injection number. VA change at 1 and 2 years following initiation of treatment were each interrogated as the dependent variable. A $p$ value $<0.05$ was considered significant.

\section{Patient and public involvement}

Patients and public were not involved in the study as this was a retrospective cohort study.

\section{Data sharing statement}

Data available from the Dryad Digital Repository: https:/ / doi.org/10.5061/dryad.97r9289

\section{RESULTS}

\section{Patient demographics}

The full data set consisted of 8174 treatment-naive eyes/6664 patients with 120756 single entries treated for neovascular AMD in the Moorfields database between 21 October 2008 and 9 August 2018.

The data set for analysis consisted of 3357 eyes/patients (61\% female). Mean age was 78 (95\% CI 77.7 to 78.3 ) years at baseline. Mean VA was $56.2(95 \%$ CI 55.6 to 56.8$)$ letters. Of these, 1105 eyes $(33 \%)$ were treated with ranibizumab, 1533 (46\%) with aflibercept, and 719 eyes (21\%) were treated with both ranibizumab and aflibercept. The starting year of treatment ranged between 2007 and 2018. Therapeutic choices at Moorfields Eye Hospital changed after 2013 and both ranibizumab and aflibercept were offered as alternative first-line agents. After this change, a number of patients were switched from one agent to another resulting in over $50 \%$ of eyes in the full dataset receiving both drugs during the course of treatment. Baseline characteristics are shown in table 1.

Of the 1162 patients not completing the 2-year follow-up date, 254 patients had died. LTFU occurred in $27 \%$ of eyes for 2 year follow-up. To address the potentially resulting survival bias of, 1 year outcomes for the cohort not completing 2-year follow-up and the cohort completing the 2-year follow-up are shown.

\section{Visual outcomes at 1 and 2 years}

Mean VA gain at 1 and 2 years were +5.5 (95\% CI 5.0 to $6.0)$ and $+4.9(95 \%$ CI 4.2 to 5.6$)$ letters, respectively. The

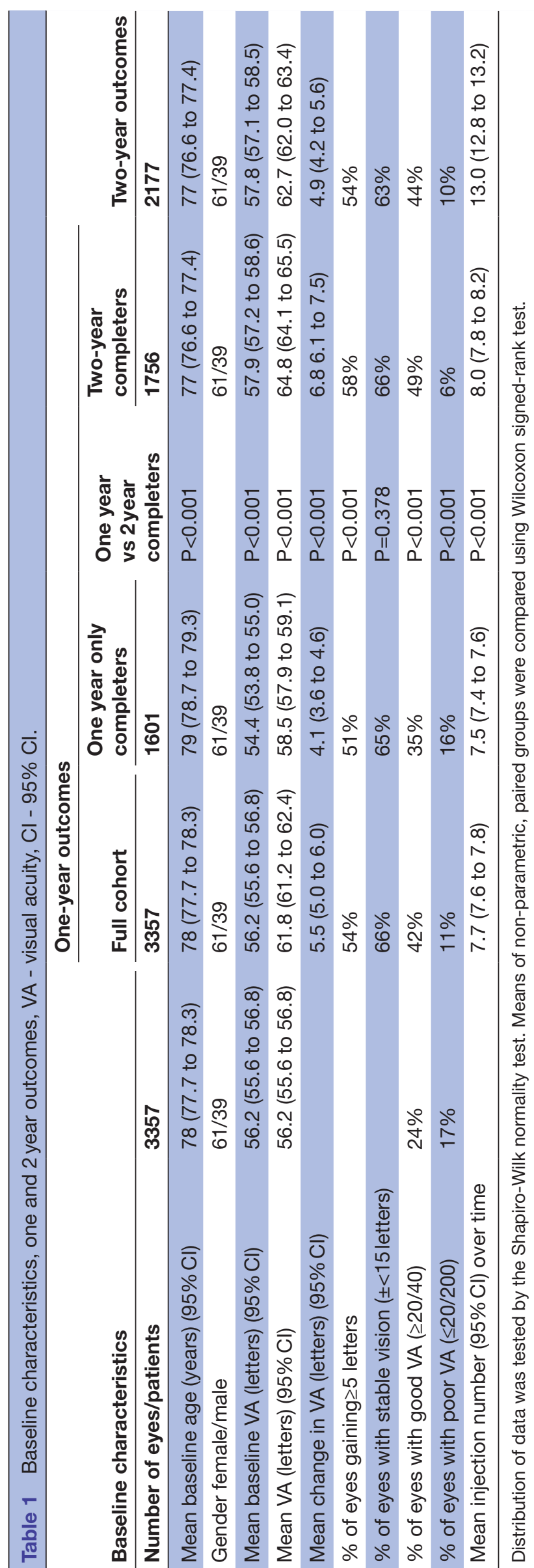



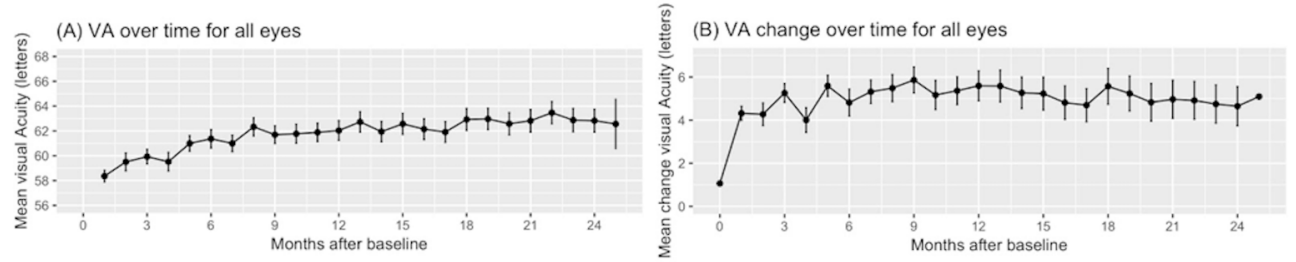

(C) VA over time stratified by follow-up period
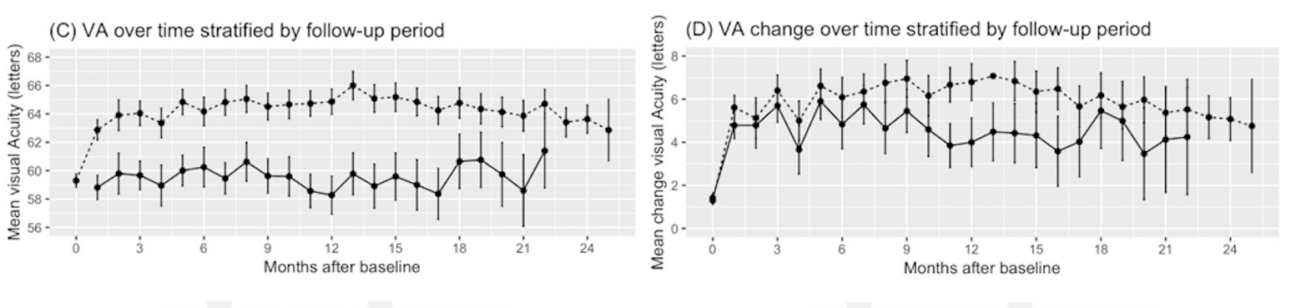

year - one year completers …. two year completers

year - one year completers …. two year completers

Figure 1 Visual acuity (A\&C) and change in visual acuity (B\&D) over time for all eyes and stratified by follow-up period (black: 1-year completers only; grey: 2-year completers). Bars represent $95 \% \mathrm{Cl}$.

mean number of injections over the first year and first 2 years were 7.7 (95\% CI 7.6 to 7.8) and 13.0 (95\% CI 12.8 to 13.2 ), respectively (figure 1 ). Percentages of eyes gaining vision (change in VA $\geq 5$ letters), stable vision (change in $\mathrm{VA}<15$ letters), good vision (VA $\geq 70$ letters/ $>20 / 40$ ), and poor vision (VA $\leq 35$ letters/ $\leq 20 / 200$ ) are shown in table 1 and figure 2.

Comparison of subgroups that did not complete the 2-year follow-up and the cohort that did complete the 2-year follow-up showed a significantly lower mean baseline VA for those with a follow-up of less than 2 years ( 54.4 vs 57.9 letters, $\mathrm{p}<0.05)$ a lower mean gain of letters ( $4.1 \mathrm{vs}$ 6.8 letters, $\mathrm{p}<0.05)$ as well as a lower injection frequency $(7.5$ vs $8.0, \mathrm{p}<0.05)$ at 1 year.

\section{Determinants of change in VA at 1 and 2 years}

Age at presentation, VA at presentation, and injection number have each been shown independently to correlate with VA outcomes eyes with neovascular AMD receiving anti-VEGF therapy. ${ }^{12}$ We therefore wanted to assess whether these parameters would correlate with 1and 2year VA outcomes in our cohort.

We carried out multiple linear regression analyses of the clinical variables (gender, baseline age, baseline VA, injection number) with VA change at 1 year (online supplementary 2, sTable 1) and 2years (online supplementary 2, sTable 2) following baseline as dependent variables. Regression models were statistically significant and suggest that a lower baseline VA, lower baseline age and higher injection number are independently associated with a higher VA change at year one and two. Indeed, this is the trend demonstrated when VA change over the observation period is stratified by baseline age and VA (figure 3).
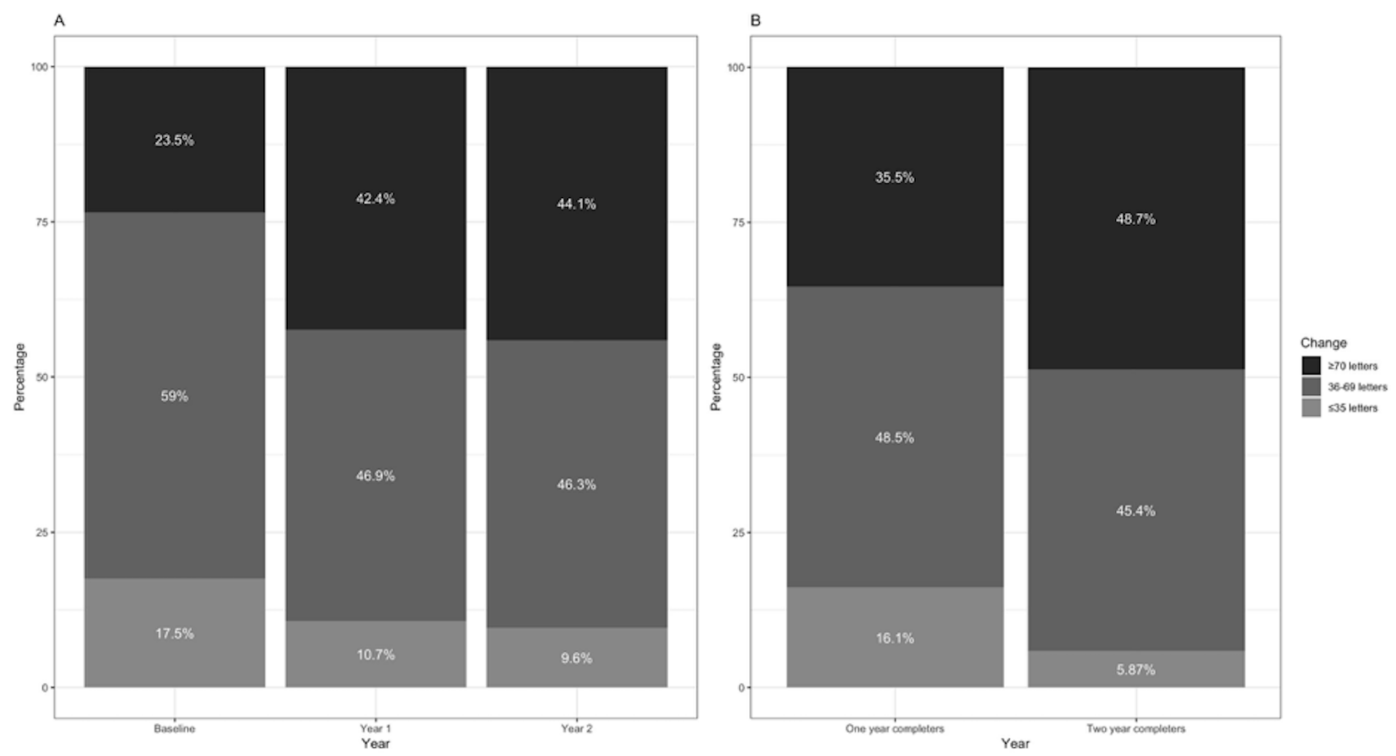

Figure 2 Percentage of eyes with good VA ( $\geq 70$ letters), intermediate VA (36-69 letters), and poor VA ( $\leq 35$ letters) at different follow-up times $(A)$ and comparison of cohorts of different follow-up times at 1 year (B). VA - visual acuity. 

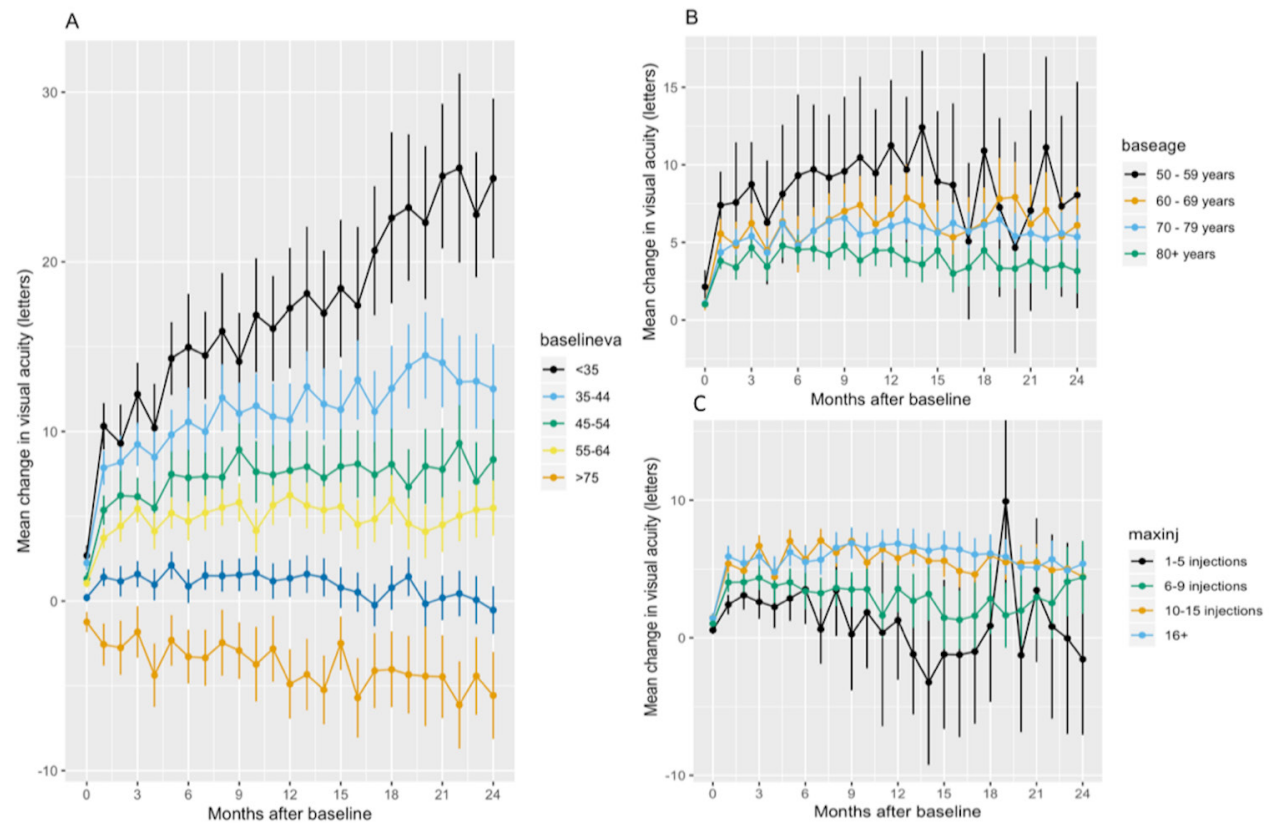

Figure 3 Change in visual acuity stratified by baseline VA (A), baseline age $(B)$ and injection number at 2 years $(\mathbf{C})$. Bars represent $95 \% \mathrm{Cl}$.

\section{DISCUSSION}

In this study, we show that patients treated with ranibizumab and/or aflibercept for neovascular AMD at Moorfields Eye Hospital achieve good visual outcomes, particularly those patients who present at an earlier age with better visual acuity, and who subsequently receive frequent intravitreal injections.

The Moorfields AMD Database is a large, consistent, and clean dataset of neovascular AMD treatment and visual outcomes, perhaps the largest single-centre dataset of its kind worldwide. We have made this freely available to download with this manuscript in an effort to benefit the AMD research community. At a minimum, this will allow for use of alternative statistical approaches and facilitate research reproducibility. ${ }^{21}$ We also hope it will allow for the testing of new hypotheses and thus provide new insights into the treatment of this condition. ${ }^{27}$ We have also developed systems so that the Moorfields AMD Database is automatically updated over time, with minimal need for manual cleaning of data. Just under 1000 new cases of neovascular AMD present to Moorfields Eye Hospital on a yearly basis - this may be particularly useful as new therapeutics for AMD continue to be introduced. Additionally, we plan on releasing data for long-term follow-up of these ( 5 years and beyond), as well as their associated raw imaging data (colour fundus photography and optical coherence tomography (OCT) in every eye at every visit).

At one and 2 years, our results of mean VA gains confirm the existing evidence in real-life studies, for example, the Fight Retinal Blindness (FRB) group in Australia/New Zealand for ranibizumab/aflibercept with nearly identical baseline characteristics and visual acuity outcomes for mixed ranibizumab/aflibercept treatment (see online supplementary 3 , sTable 3$).{ }^{10} 28$

However, VA gains reported by the Writing Committee for the UK Age-Related Macular Degeneration EMR Users Group are considerably poorer which likely is explained by the reported capacity constraints resulting in reduced treatment frequency of with a mean of 9.4 injections over 2 years versus over 13 in our cohort and the FRB. ${ }^{16}$ VA results from randomised prospective studies (eg, the Comparison of Age-related macular degeneration Treatment Trials (CATT) and Vascular endothelial growth factor Trap-Eye: Investigation of Efficacy and Safety in Wet AMD study (VIEW) ) have been shown to be superior to retrospective real-life data. ${ }^{14}$

This is also reflected in our data and is explained by the broader inclusion criteria, and the less strict treatment regimens with fewer administered injections. Comparison of cohorts that completed only 1 year of follow-up versus 2 or more years showed that eyes with shorter follow-up were older, had lower baseline VA, gained fewer letters at the 1 year follow-up, and received fewer injections over the first year. The loss to follow-up reflects the real-life setting of the study where patients transfer to stable AMD clinics, their vision has deteriorated and rendered further treatment unreasonable, or they are not able to further attend clinics. We deliberately did not perform any imputational replacement of missing data, but clearly describe the baseline characteristics and compare the 1-year results of the cohort LTFU before 2 years. ${ }^{12}$

VA gain over time is dependent on baseline characteristics and injection frequency. ${ }^{12} 1429$ Increasing age diminishes the VA gain expected as does a higher baseline acuity due to ceiling effect. ${ }^{30}$ Baseline VA could even 
emerge as a surrogate measure for accessibility to treatment and quality of care, since simply looking at VA gains would underestimate centres that achieve short time from diagnosis to first treatment resulting in above average baseline VA but ceiling effect on VA gains. ${ }^{812} 16$ Injection frequency has been recognised as another significant factor influencing VA gain and has been hypothesised to be the major factor in studies comparing ranibizumab and aflibercept due to the change in posology from treatment as needed to treat-and-extend concomitant with the change from ranibizumab to aflibercept in clinical practice. $^{14293132}$.

The retrospective nature and EMR-based data collection of our study introduce several limiting factors. Smoking status of our patients was not consistently available and thus, could not be included in the prediction model. Smoking has been identified as a risk factor for the development of neovascular AMD, but might also impact treatment response. ${ }^{33}$ There is invariably survival bias within the data, as LTFU cannot be assumed to occur at random. However, baseline characteristics of LTFU as well as differences in outcomes for 1- and 2-year follow-up cohorts have been clearly described to address this. To date, there is no systematic collection of patient-reported outcome measures (mobility and independence, emotional wellbeing, as well as reading and accessing information questionnaires) as suggested by ICHOM. ${ }^{22}$ EMR studies introduce new challenges to medical research: Data quality issues, hidden in large datasets, could lead to false interpretation, i.e. 'garbage in - garbage out', lack of computer science skills may limit reproducibility of research results, and sharing of medical data poses legal issues. ${ }^{12} 202134$ Our study addresses this with a transparent, STROBE statement conforming structure, and an open science approach with information governance approved depersonalised data sharing.

The main advantages of this study are the quality and amount of data coming from one single centre and one database. Moorfields Eye Hospital has a standardised treatment protocol for neovascular AMD, formerly treatment as needed, and fixed-first year/treat-and extend regimen with the introduction of aflibercept in 2014 (flow chart for aflibercept use is shown in online supplementary 4, sFigure 2). The extensive manual cleaning and the homogeneous standards of data input (VA in ETDRS letters, mandatory fields) have formed a highly reliable resource which will be enhanced in the future with an automated update and validation to allow for continued growth and quality improvement of clinical AMD data.

In conclusion, this study shows that with a diligent approach, analysis of well maintained EMR data can lead to high quality real-life results and electronic availability of data facilitates maximisation of its potential in sharing research resources with the community, ultimately with the goal of improving patient care in real-life. In the near future, we plan to report on long-term visual outcomes (eg, after 5years), anatomic outcomes, and fellow-eye involvement, as well as the differential therapeutic effects of ranibizumab and aflibercept. In each case, we plan to release the raw data that underpins these reports - we hope that this will help promote an open-science approach to the study of neovascular AMD, and thus to direct patient benefit in the longer term.

\section{Author affiliations}

${ }^{1} \mathrm{NIHR}$ Biomedical Research Centre, Moorfields Eye Hospital NHS Foundation Trust, London, UK

${ }^{2}$ Department of Ophthalmology, UniversitatsSpital Zurich, Zurich, Switzerland ${ }^{3}$ Augenklinik, Klinikum der Universitat Munchen, Munchen, Bayern, Germany ${ }^{4}$ Augenklinik, Luzerner Kantonsspital Zentrumsspital, Luzern, Switzerland ${ }^{5}$ Department of Ophthalmology, University of Washington, Seattle, Washington, USA ${ }^{6}$ School of Biological Sciences, University of Manchester, Manchester, UK

Contributors KF has drafted the manuscript and contributed to data acquisition, analysis, and interpretation of data. She is accountable for all aspects of the work and has approved for the final version to be published. SW and KUK have contributed in design of the study, interpretation of data as well as critical revision of the manuscript. They share accountability for all aspects of the work and have approved for the final version to be published. LF, DJF, and AYL have contributed to analysis and interpretation of the data and critical revision of the manuscript. They share accountability for all aspects of the work and have approved for the final version to be published. GM, GP, RC, and NP have contributed to acquisition of data and critical revision of the manuscript. They share accountability for all aspects of the work and have approved for the final version to be published. KB, PP, AT, and PAK have contributed to conception of the work, interpretation of data and critical revision of the manuscript. They share accountability for all aspects of the work and have approved for the final version to be published.

Funding The research supported by the National Institute for Health Research (NIHR) Biomedical Research Centre based at Moorfields Eye Hospital NHS Foundation Trust and UCL Institute of Ophthalmology. The views expressed are those of the author(s) and not necessarily those of the NHS, the NIHR or the Department of Health. Dr. Fasler has received fellowship support from Alfred Vogt Stipendium and Schweizerischer Fonds zur Verhütung und Bekämpfung der Blindheit.

\section{Competing interests None declared.}

Patient consent for publication Not required.

Ethics approval Institutional Review Board at Moorfields (ROAD17/031).

Provenance and peer review Not commissioned; externally peer reviewed.

Data sharing statement Depersonalised data for this study will be openly available from the Dryad Digital Repository https://doi.org/10.5061/dryad.97r9289. Depersonalisation was carried out through hash function anonymisation of patient identification numbers, replacement of appointment dates with follow-up days to baseline, and categorising extreme age groups into age categories. Approval of adequate depersonalisation was obtained by Moorfields Information Governance

Open access This is an open access article distributed in accordance with the Creative Commons Attribution Non Commercial (CC BY-NC 4.0) license, which permits others to distribute, remix, adapt, build upon this work non-commercially, and license their derivative works on different terms, provided the original work is properly cited, appropriate credit is given, any changes made indicated, and the use is non-commercial. See: http://creativecommons.org/licenses/by-nc/4.0/.

\section{REFERENCES}

1. Martin DF, Maguire MG, Fine SL, et al. Ranibizumab and bevacizumab for treatment of neovascular age-related macular degeneration: two-year results. Ophthalmology 2012;119:1388-98.

2. Brown DM, Michels M, Kaiser PK, et al. Ranibizumab versus verteporfin photodynamic therapy for neovascular age-related macular degeneration: Two-year results of the ANCHOR study. Ophthalmology 2009;116:57-65.

3. Rosenfeld PJ, Brown DM, Heier JS, et al. Ranibizumab for neovascular age-related macular degeneration. $N$ Engl J Med 2006;355:1419-31

4. Heier JS, Brown DM, Chong V, et al. Intravitreal aflibercept (VEGF trap-eye) in wet age-related macular degeneration. Ophthalmology 2012;119:2537-48. 
5. Holz FG, Tadayoni R, Beatty S, et al. Multi-country real-life experience of anti-vascular endothelial growth factor therapy for wet age-related macular degeneration. Br J Ophthalmol 2015;99:220-6.

6. Cohen SY, Mimoun G, Oubraham H, et al. Changes in visual acuity in patients with wet age-related macular degeneration treated with intravitreal ranibizumab in daily clinical practice: the LUMIERE study. Retina 2013;33:474-81.

7. Finger RP, Wiedemann P, Blumhagen F, et al. Treatment patterns, visual acuity and quality-of-life outcomes of the WAVE study - a noninterventional study of ranibizumab treatment for neovascular age-related macular degeneration in Germany. Acta Ophthalmol 2013;91:540-6.

8. Liew G, Lee AY, Zarranz-Ventura J, et al. The UK Neovascular AMD Database Report 3: inter-centre variation in visual acuity outcomes and establishing real-world measures of care. Eye 2016;30:1462-8.

9. Eleftheriadou M, Gemenetzi M, Lukic M, et al. Three-Year Outcomes of Aflibercept Treatment for Neovascular Age-Related Macular Degeneration: Evidence from a Clinical Setting. Ophthalmol Ther 2018;7:361-8.

10. Barthelmes D, Nguyen V, Daien V, et al. Two year outcomes of "treat and extend" intravitreal therapy using aflibercept preferentially for neovascular age-related macular degeneration. Retina 2018;38:20-8.

11. Chong V. Ranibizumab for the treatment of wet AMD: a summary of real-world studies. Eye 2016;30:270-86.

12. Mehta H, Tufail A, Daien V, et al. Real-world outcomes in patients with neovascular age-related macular degeneration treated with intravitreal vascular endothelial growth factor inhibitors. Prog Retin Eye Res 2018:65:127-46.

13. Lotery A, Griner R, Ferreira A, et al. Real-world visual acuity outcomes between ranibizumab and aflibercept in treatment of neovascular AMD in a large US data set. Eye 2017;31:1697-706.

14. Rao P, Lum F, Wood K, et al. Real-World Vision in Age-Related Macular Degeneration Patients Treated with Single Anti-VEGF Drug Type for 1 Year in the IRIS Registry. Ophthalmology 2018;125.

15. Almuhtaseb H, Johnston RL, Talks JS, et al. Second-year visual acuity outcomes of nAMD patients treated with aflibercept: data analysis from the UK Aflibercept Users Group. Eye 2017;31:1582-8.

16. Writing Committee for the UK Age-Related Macular Degeneration EMR Users Group. The neovascular age-related macular degeneration database: multicenter study of 92976 ranibizumab injections: report 1: visual acuity. Ophthalmology 2014;121:1092-101.

17. Kataja M, Hujanen $\mathrm{P}$, Huhtala $\mathrm{H}$, et al. Outcome of anti-vascular endothelial growth factor therapy for neovascular age-related macular degeneration in real-life setting. Br J Ophthalmol 2018;102:959-65.

18. Ozturk M, Harris ML, Nguyen V, et al. Real-world visual outcomes in patients with neovascular age-related macular degeneration receiving aflibercept at fixed intervals as per UK licence. Clin Exp Ophthalmol 2018;46.

19. Denaxas S, Direk K, Gonzalez-Izquierdo A, et al. Methods for enhancing the reproducibility of biomedical research findings using electronic health records. BioData Min 2017;10:31.

20. von Elm E, Altman DG, Egger M, et al. The Strengthening the Reporting of Observational Studies in Epidemiology (STROBE) statement: guidelines for reporting observational studies. Lancet 2007;370:1453-7.

21. Benchimol El, Smeeth L, Guttmann A, et al. The REporting of studies Conducted using Observational Routinely-collected health Data (RECORD) statement. PLoS Med 2015;12:e1001885.

22. Rodrigues IA, Sprinkhuizen SM, Barthelmes D, et al. Defining a Minimum Set of Standardized Patient-centered Outcome Measures for Macular Degeneration. Am J Ophthalmol 2016;168:1-12.

23. Packer M. Data sharing in medical research. BMJ 2018;360:k510.

24. Cohen D. Why have UK doctors been deterred from prescribing Avastin? BMJ 2015;350:h1654

25. Hambleton D. Commentary: NHS patients should have a choice of drug for wet age-related macular degeneration, despite pressure from pharma. BMJ 2017;359:j5013.

26. Lange C, Feltgen N, Junker B, et al. Resolving the clinical acuity categories "hand motion" and "counting fingers" using the Freiburg Visual Acuity Test (FrACT). Graefes Arch Clin Exp Ophthalmol 2009;247:137-42.

27. RPB/Academy Award. American Academy of Ophthalmology. 2017. https://www.aao.org/iris-registry/data-analysis/research-to-preventblindness-research-grants (cited 24 Jun 2018).

28. Arnold JJ, Campain A, Barthelmes D, et al. Two-year outcomes of "treat and extend" intravitreal therapy for neovascular age-related macular degeneration. Ophthalmology 2015;122:1212-9.

29. Kim LN, Mehta H, Barthelmes D, et al. Metaanalysis of real-world outcomes of intravitreal ranibizumab for the treatment of neovascular age-related macular degeneration. Retina 2016;36:1418-31.

30. Holz FG, Tadayoni R, Beatty S, et al. Key drivers of visual acuity gains in neovascular age-related macular degeneration in real life: findings from the AURA study. Br J Ophthalmol 2016;100:1623-8.

31. Sarwar S, Clearfield E, Soliman MK, et al. Aflibercept for neovascular age-related macular degeneration. Cochrane Database Syst Rev 2016:2:CD011346.

32. Lee AY, Lee CS, Egan CA, et al. UK AMD/DR EMR REPORT IX: comparative effectiveness of predominantly as needed (PRN) ranibizumab versus continuous aflibercept in UK clinical practice. $\mathrm{Br}$ J Ophthalmol 2017;101:1683-8.

33. Velilla S, García-Medina JJ, García-Layana A, et al. Smoking and age-related macular degeneration: review and update. J Ophthalmol 2013;89.

34. Kilkenny MF, Robinson KM. Data quality: "Garbage in - garbage out". Health Inf Manag 2018;47:103-5. 